\title{
Dual parameterization and Abel transform tomography for twist-3 DVCS
}

\author{
Alena M. Moiseeva ${ }^{a, b}$ and Maxim V. Polyakov ${ }^{b, c}$ \\ ${ }^{a}$ Bogoliubov Laboratory of Theoretical Physics, JINR, 141980, Moscow Region, Dubna, \\ Russia \\ ${ }^{b}$ Institut für Theoretische Physik II, Ruhr-Universität Bochum, D-44780 Bochum, \\ Germany \\ ${ }^{c}$ Petersburg Nuclear Physics Institute, Gatchina, St. Petersburg 188350, Russia
}

\begin{abstract}
We derive dual parameterization for the DVCS amplitude to the twist-3 accuracy. The relation of the dual parameterization to the Abel transform tomography for amplitudes of hard exclusive processes is elaborated. We prove dispersion relations for the twist-3 DVCS amplitude in the Wandzura - Wilczek approximation and show that the subtraction constant is given by the D-term form factor. The same as in the case of twist-2 DVCS amplitude.
\end{abstract}

\section{Dual parameterization facilitates Abel tomography for DVCS amplitudes}

The dual parameterization [1] of generalized parton distributions (GPDs) [2] (for recent reviews of GPDs see [3, 4, 5, 6]) provides us handy and flexible tool to describe amplitudes of hard exclusive processes in terms of single function - so-called quintessence function. This function quantifies the maximum amount of information about GPDs, which can be extracted from data on hard exclusive processes. First phenomenological applications of this parameterization to DVCS give very promising results [7, 8]. In present paper we extend the dual parameterization to the twist-3 GPDs in the Wandzura-Wilczek approximation.

Let us first remind basics of the dual parameterization and dwell on its relation to Abel tomography [9]. The leading order twist-2 amplitude of hard exclusive reactions is expressed in terms of the following elementary amplitude*:

$$
A^{\mathrm{tw} 2}(\xi, t)=\int_{0}^{1} d x H(x, \xi, t)\left[\frac{1}{\xi-x-i 0}-\frac{1}{\xi+x-i 0}\right] .
$$

We see that the amplitude is given by the convolution integral in which dependence of GPDs on variable $x$ is "integrated out". One can not completely restore the GPD $H(x, \xi, t)$ from Eq. (11), because from this equation one can obtain the same amplitude with different GPDs $H(x, \xi, t)$. In dual parameterization the expression for the amplitude has the form

${ }^{*}$ As the first step we restrict ourselves to DVCS on spinless hadron. 
[1, 10, 11]:

$$
\begin{aligned}
\operatorname{Im} A^{\mathrm{tw} 2}(\xi, t) & =\int_{\frac{1-\sqrt{1-\xi^{2}}}{\xi}}^{1} \frac{d x}{x} N(x, t)\left[\frac{1}{\sqrt{\frac{2 x}{\xi}-x^{2}-1}}\right] \\
\operatorname{Re} A^{\mathrm{tw} 2}(\xi, t) & =\int_{0}^{\frac{1-\sqrt{1-\xi^{2}}}{\xi}} \frac{d x}{x} N(x, t)\left[\frac{1}{\sqrt{1-\frac{2 x}{\xi}+x^{2}}}+\frac{1}{\sqrt{1+\frac{2 x}{\xi}+x^{2}}}-\frac{2}{\sqrt{1+x^{2}}}\right] \\
& +\int_{\frac{1-\sqrt{1-\xi^{2}}}{\xi}}^{1} \frac{d x}{x} N(x, t)\left[\frac{1}{\sqrt{1+\frac{2 x}{\xi}+x^{2}}}-\frac{2}{\sqrt{1+x^{2}}}\right]+2 D(t) .
\end{aligned}
$$

The amplitude is completely determined by, so-called, GPD-quintessence function $N(x, t)$ [8] and by the D-form factor $D(t)$. The latter is :

$$
D(t)=\sum_{n=1}^{\infty} d_{n}(t)=\frac{1}{2} \int_{-1}^{1} d z \frac{D(z, t)}{1-z},
$$

where $D(z, t)$ is the D-term [12]. One can check [10] that the amplitude given by Eqs. (2, 3]) automatically satisfies the dispersion relation with the subtraction constant given by the D- form factor, as it should be on general grounds [13, 14]. The GPD-quintessence function $N(x, t)$ is expressed in terms of set of forward-like function $\dagger$ as:

$$
N(x, t)=\sum_{\nu=0}^{\infty} x^{2 \nu} Q_{2 \nu}(x, t) .
$$

The knowledge of the LO amplitude is in one-to-one equivalence to the knowledge of the function $N(x, t)$ and the D-form factor $D(t)$, because the Eq. (2) can be inverted [10], i.e. the function $N(x, t)$ can be expressed unambiguously in terms of amplitude. As the function $N(x, t)$ is unambiguously restored from the amplitude it means that it contains maximal information about GPDs which one can, in principle, obtain from the amplitudes.

$\dagger$ We call the functions $Q_{2 \nu}(x, t)$ forward-like because [10]:

- At the LO scale dependence of functions $Q_{2 \nu}(x, t)$ is given by the standard DGLAP evolution equation, so that these functions behave as usual parton distributions under QCD evolution.

- The function $Q_{0}(x, t)$ is related to the forward $t$-dependent quark densities $q(x, t)$ (at $t=0$ they are reduced to usual parton densities $q(x)$ measured in DIS) as:

$$
Q_{0}(x, t)=q(x, t)+\bar{q}(x, t)-\frac{x}{2} \int_{x}^{1} \frac{d z}{z^{2}}(q(z, t)+\bar{q}(z, t)) .
$$

- The expansion of the GPD $H(x, \xi, t)$ around the point $\xi=0$ with fixed $x$ to the order $\xi^{2 \nu}$ involves only finite number of functions $Q_{2 \mu}(x, t)$ with $\mu \leq \nu$.

See original papers [1, 10, 11] for detailed discussion of properties of these functions and their physics interpretation. 
This inversion, actually, corresponds to the Abel transform tomography [9]. Let us show relation of Eq. (2) to tomography. It is useful to make the following substitution for the integration variable $x$ in Eq. (21):

$$
\frac{1}{w}=\frac{1}{2}\left(x+\frac{1}{x}\right)
$$

This substitution correspond to famous Joukowskii conformal map [16], which historically was used to understand some principles of aerofoil design. After this change of variables the expression for the imaginary part of the amplitude gets the form:

$$
\operatorname{Im} A^{\mathrm{tw} 2}(\xi, t)=\int_{\xi}^{1} \frac{d w}{w} M(w, t) \frac{\sqrt{\xi}}{\sqrt{w-\xi}},
$$

where the function $M(w, t)$ is related to the function $N(x, t)$ as:

$$
M(w, t)=N\left(\frac{1-\sqrt{1-w^{2}}}{w}, t\right) \frac{w}{\sqrt{2\left(1-w^{2}\right)} \sqrt{1-\sqrt{1-w^{2}}}} .
$$

The Eq. (7) is typical for the Abel tomography. In two dimensions, the Abel transform $a(y)$ can be interpreted as the projection of a axially symmetric function $m(\rho)$ along a set of parallel lines of sight which are at distance $y$ from the origin. Referring to Fig. 1, the observer see the image:

$$
a(y)=\int_{-\infty}^{\infty} d x m(\rho) .
$$

Now, using axial symmetry of the function $m(\rho)$, we make substitution for the integration variable $x^{2}=\rho^{2}-y^{2}$, as the result we obtain the following expression for the image function $a(y)$ :

$$
a(y)=\int_{y^{2}}^{\infty} d \rho^{2} \frac{m(\rho)}{\sqrt{\rho^{2}-y^{2}}} .
$$

This equation has exactly the form (with obvious identification of variables and functions) of Eq. (7). We see that we reduce our problem about GPDs to the restoring of images of axially symmetric objects from their photographs on plane. This problem is solved unambiguously with help of Abel transform [9]. Let us demonstrate us this directly for Eq. (7). Introducing the following notations $m(w, t)=\frac{M(w, t)}{w}$ and $a(\xi, t)=\frac{1}{\sqrt{\xi}} \operatorname{Im} A(\xi, t)$ $\left(\rho^{2}=w\right.$ and $\left.y^{2}=\xi\right)$, we arrive at the integral equation of Abel:

$$
a(\xi, t)=\int_{\xi}^{1} d w \frac{m(w, t)}{\sqrt{w-\xi}},
$$

\footnotetext{
$\ddagger$ See Ref. [15] for the pioneering applications of the Radon tomography methods to GPDs.

$\S$ It would be interesting to understand the deep meaning of this axial symmetry in context of GPD problem. Amplitudes of hard exclusive processes are photographs of some object which is axial symmetric in plane of variables $X=\sqrt{\frac{2 x}{1+x^{2}}-\xi}$ and $Y=\sqrt{\xi}$.
} 


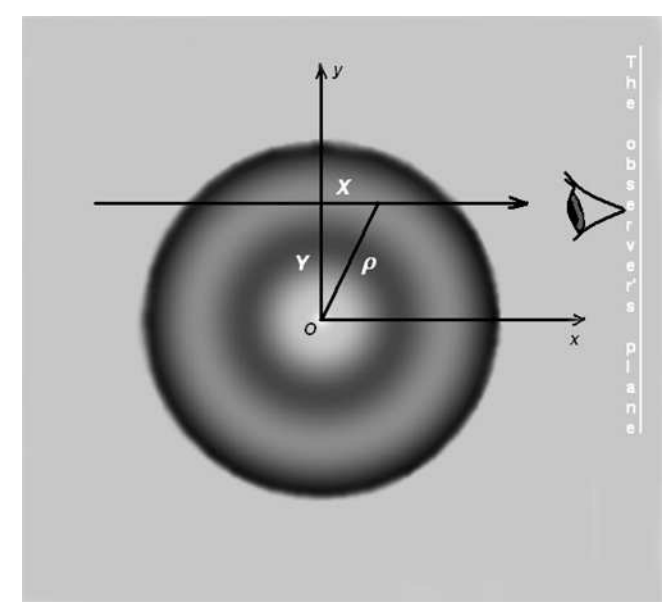

Figure 1: Illustration for Abel transform tomography

which has very simple and unambiguous solution. Indeed, to solve the Abel equation we multiply both sides of the above equation equality by $1 / \sqrt{\xi-z}$ and integrate it over $\xi$ from $z$ to 1 . Then changing sequence of integration over $\xi$ and $w$, we obtain

$$
\begin{gathered}
\int_{z}^{1} d \xi \frac{a(\xi, t)}{\sqrt{\xi-z}}=\int_{z}^{1} d \xi \frac{1}{\sqrt{\xi-z}} \int_{\xi}^{1} d w \frac{m(w, t)}{\sqrt{w-\xi}}=\int_{z}^{1} d w m(w, t) \int_{z}^{w} d \xi \frac{1}{\sqrt{(w-\xi)(\xi-z)}} \\
\int_{z}^{1} d \xi \frac{a(\xi, t)}{\sqrt{\xi-z}}=\pi \int_{z}^{1} d w m(w, t) .
\end{gathered}
$$

Now differentiating that expression over $z$, one can write

$$
m(w, t)=-\frac{1}{\pi} \frac{d}{d w} \int_{w}^{1} d \xi \frac{a(\xi, t)}{\sqrt{\xi-w}}
$$

This solves the Abel tomography problem. Making necessary trivial change of variables we obtain the expression for the GPD- quintessence function $N(x, t)$ in terms of the amplitude [10]:

$$
N(x, t)=\frac{2}{\pi} \frac{x\left(1-x^{2}\right)}{\left(1+x^{2}\right)^{3 / 2}} \int_{\frac{2 x}{1+x^{2}}}^{1} \frac{d \xi}{\xi^{3 / 2}} \frac{1}{\sqrt{\xi-\frac{2 x}{1+x^{2}}}}\left\{\frac{1}{2} \operatorname{Im} A^{\mathrm{tw} 2}(\xi, t)-\xi \frac{d}{d \xi} \operatorname{Im} A^{\mathrm{tw} 2}(\xi, t)\right\}
$$

The beauty of the Abel transform lies in its exactness and conciseness. Many effective numerical methods to implement the Abel transform were developed, see e.g [17]. For instance, Chan and Lu [18] suggested the form of Abel transform which avoids derivatives (which can be noisy when applied to data) of the "image function" ( $\operatorname{Im} A$ in our case). Our Eq. (11) in representation of Chan and Lu has the form: 


$$
\begin{aligned}
N(x, t) & =-\frac{1}{\pi} \frac{x\left(1-x^{2}\right)}{\left(1+x^{2}\right)^{3 / 2}} \int_{\frac{2 x}{1+x^{2}}}^{1} d \xi \frac{1}{\left(\xi-\frac{2 x}{1+x^{2}}\right)^{\frac{3}{2}}} \\
& \times\left\{\frac{1}{\sqrt{\xi}} \operatorname{Im} A^{\mathrm{tw} 2}(\xi, t)-\sqrt{\frac{1+x^{2}}{2 x}} \operatorname{Im} A^{\mathrm{tw} 2}\left(\frac{2 x}{1+x^{2}}, t\right)\right\} \\
& +\frac{1}{\pi} \frac{\sqrt{2 x}(1+x)}{\sqrt{1+x^{2}}} \operatorname{Im} A^{\mathrm{tw} 2}\left(\frac{2 x}{1+x^{2}}, t\right) .
\end{aligned}
$$

\section{Dual parameterization for twist-3 DVCS amplitude}

The DVCS twist-3 amplitude was computed in Refs. [19, 20, 21, 22, it includes the contributions which are suppressed by power of $1 / Q$ relative to the twist- 2 amplitude. The inclusion of such terms is mandatory to ensure the electromagnetic gauge invariance of the DVCS amplitude to the order $\sqrt{-t} / Q$, also these contributions can give sizable contributions to the DVCS observables [23]. At the order $1 / Q$ the DVCS amplitude depends on a set of new GPDs. In Refs. [21, 25, 22, 27] it was shown in that in the socalled Wandzura-Wilczek (WW) 24] approximation these new functions can be expressed in terms of twist-2 GPDs. The WW approximation corresponds to neglecting the nucleon matrix elements of the 'genuine twist-3' operators of the type $\bar{\psi} G \psi$, i.e. neglecting the nonperturbative quark-gluon correlations in the nucleon. In principle this approximation is not justified by any small parameter of the theory. However, recent measurements of the polarized structure function $g_{T}(x)$ [29] show that the WW approximation works pretty well in this case. Also the estimates of the 'genuine twist-3' contributions polarized structure functions $g_{T}(x)$ and $h_{L}(x)$ [30, 31, and to twist-3 GPDs 32 in the theory of instanton vacuum (which is a model of non-perturbative quark-gluon correlations) showed that these contributions are parametrically suppressed relative to the 'kinematical' part of the twist-3 contributions by the packing fraction of instantons in the vacuum.

To the twist-3 accuracy the DVCS amplitude has the form:

$$
\begin{aligned}
T^{\mu \nu} & =\frac{1}{2 P \cdot Q} \int d x\left(\frac{1}{\xi-x-i 0}-\frac{1}{\xi+x-i 0}\right)\left[H ( x , \xi ) \left(-2 \xi P^{\mu} P^{\nu}-P^{\mu} Q^{\nu}-P^{\nu} Q^{\mu}\right.\right. \\
& \left.+g^{\mu \nu}(P \cdot Q)-\frac{1}{2} P^{\mu} \Delta_{\perp}^{\nu}+\frac{1}{2} P^{\nu} \Delta_{\perp}^{\mu}\right) \\
& \left.-\left[H_{3}(x, \xi)+\frac{\xi}{x} H_{A}(x, \xi)\right] \Delta_{\perp}^{\nu}\left(3 \xi P^{\mu}+Q^{\mu}\right)\right]
\end{aligned}
$$

where $P=\left(p+p^{\prime}\right) / 2, Q=\left(q+q^{\prime}\right) / 2, \Delta=q-q^{\prime}=p^{\prime}-p=-2 \xi P+\Delta_{\perp}$, while $p, p^{\prime}, q, q^{\prime}$ are the initial and final momenta of pion and photon, respectively. The three terms in the square brackets are individually gauge invariant up to order $\Delta_{\perp}^{2}$, i.e. $q_{\nu}^{\prime} T^{\mu \nu}=O\left(\Delta_{\perp}^{2}\right)=$ $q_{\mu} T^{\mu \nu}$. As $P$ and $Q$ are longitudinal and $\Delta_{\perp}$ transverse, the first term corresponds to 
transverse polarization of the virtual photon, whereas the second term to the longitudinally polarized virtual photon The second term contains the new twist-3 contributions to the DVCS amplitude, which are defined as:

$$
\begin{aligned}
\int \frac{d \lambda}{2 \pi} e^{i \lambda x(\bar{P} n)}\left\langle p^{\prime}\left|\bar{\psi}\left(-\frac{\lambda}{2} n\right) \gamma^{\mu} \psi\left(\frac{\lambda}{2} n\right)\right| p\right\rangle & =P^{\mu} H(x, \xi)+\Delta_{\perp}^{\mu} H_{3}(x, \xi), \\
\int \frac{d \lambda}{2 \pi} e^{i \lambda x(\bar{P} n)}\left\langle p^{\prime}\left|\bar{\psi}\left(-\frac{\lambda}{2} n\right) \gamma^{\mu} \gamma_{5} \psi\left(\frac{\lambda}{2} n\right)\right| p\right\rangle & =i \varepsilon_{\mu \alpha \beta \delta} \Delta^{\alpha} P^{\beta} n^{\delta} H_{A}(x, \xi) .
\end{aligned}
$$

Here the light-cone vector $n$ is normalized as $n \cdot P=1$

We introduce elementary twist-3 amplitude (in complete analogy with twist-2 that (1)), which corresponds to longitudinally polarized virtual photon:

$$
A^{\mathrm{tw} 3}(\xi, t)=-\frac{\xi}{2} \int_{0}^{1} d x\left(\frac{1}{\xi-x-i 0}-\frac{1}{\xi+x-i 0}\right) 4\left[H_{3}(x, \xi, t)+\frac{\xi}{x} H_{A}(x, \xi, t)\right] .
$$

This amplitude can be extracted from the DVCS data considering azimuthal angle dependence of the observables.

For the new twist-3 GPDs we use WW approximation [25, 26]:

$$
\begin{aligned}
4\left[H_{3}(x, \xi, t)+\frac{\xi}{x} H_{A}(x, \xi, t)\right] & =\frac{x-\xi}{x}\left\{\theta(\xi-x) \int_{-1}^{x} \frac{d y}{y-\xi} \partial_{+} H-\theta(x-\xi) \int_{x}^{1} \frac{d y}{y-\xi} \partial_{+} H\right\} \\
& +\frac{x+\xi}{x}\left\{\theta(-x-\xi) \int_{-1}^{x} \frac{d y}{y+\xi} \partial_{-} H-\theta(x+\xi) \int_{x}^{1} \frac{d y}{y+\xi} \partial_{-} H\right\}
\end{aligned}
$$

where

$$
\partial_{ \pm} H=\frac{\partial H(x, \xi, t)}{\partial \xi} \pm \frac{\partial H(x, \xi, t)}{\partial x} .
$$

After substitution of this expression into Eq. (16) we obtain the expression for the twist-3 amplitude in terms of twist-2 GPD $H(x, \xi, t)$ :

$$
A^{\mathrm{tw} 3}(\xi, t)=\frac{\xi}{2} \int_{0}^{1} d x \frac{2 x}{x^{2}-\xi^{2}}\left[\theta(\xi-x) \frac{x-\xi}{x} \int_{-1}^{x} \frac{d y}{y-\xi} \partial_{+} H-\theta(x+\xi) \frac{x+\xi}{x} \int_{x}^{1} \frac{d y}{y+\xi} \partial_{-} H\right] \text {. (17 }
$$

To compute the twist- 3 amplitude we employ formal series decomposition of twist2 GPD $H(x, \xi, t)$, which represents GPDs as the infinite sum of t-channel resonance exchanges [28]:

$$
H(x, \xi, t)=\sum_{n=1,3 \ldots}^{\infty} \sum_{l=0,2 \ldots}^{n+1} B_{n l}(t) \theta\left(1-\frac{x^{2}}{\xi^{2}}\right)\left(1-\frac{x^{2}}{\xi^{2}}\right) C_{n}^{3 / 2}\left(\frac{x}{\xi}\right) P_{l}\left(\frac{1}{\xi}\right)
$$

It is easy to see that

$$
\begin{aligned}
\partial_{ \pm} H(y, \xi, t) & =-\left(\frac{y}{\xi} \mp 1\right) \frac{\partial H(y, \xi, t)}{\partial y} \\
& +\sum_{n=1,3 \ldots}^{\infty} \sum_{l=0,2 \ldots}^{n+1} B_{n l}(t) \theta\left(1-\frac{x^{2}}{\xi^{2}}\right)\left(1-\frac{x^{2}}{\xi^{2}}\right) C_{n}^{3 / 2}\left(\frac{x}{\xi}\right) \frac{\partial P_{l}\left(\frac{1}{\xi}\right)}{\partial \xi} .
\end{aligned}
$$


Substituting this expression into Eq. (17) we obtain the partial wave representation of the twist-3 amplitude:

$$
A^{\mathrm{tw} 3}(\xi, t)=A^{\mathrm{tw} 2}(\xi, t)+2 \sum_{n=1,3 \ldots}^{\infty} \sum_{l=0,2 \ldots}^{n+1} B_{n l}(t) \xi \frac{\partial P_{l}\left(\frac{1}{\xi}\right)}{\partial \xi} \frac{1}{(n+1)(n+2)} .
$$

Here $A^{\text {tw2 }}$ is the twist-2 DVCS amplitude given by Eqs. (2),31), so that the new ingredient is the second term in above expression. For that term we introduce the following notation:

$$
A_{+}=2 \sum_{n=1,3 \ldots}^{\infty} \sum_{l=0,2 \ldots}^{n+1} B_{n l}(t) \xi \frac{\partial P_{l}\left(\frac{1}{\xi}\right)}{\partial \xi} \frac{1}{(n+1)(n+2)}
$$

and perform resummation of partial waves for it. To perform this resummation we, following Ref. [1], represent the coefficients $B_{n l}(t)$ as the Mellin moments of forward-like functions $Q_{2 \nu}(x, t)$ :

$$
B_{n, n+1-2 \nu}(t)=\int_{0}^{1} d x x^{n} Q_{2 \nu}(x, t) .
$$

Substituting this equation to (21) changing sequence of summation and integration we obtain:

$$
A_{+}(\xi, t)=2 \sum_{\nu=0}^{\infty} \xi \frac{\partial}{\partial \xi} \int_{0}^{1} \frac{d x}{x} x^{k} S_{2 \nu}(x, t) \sum_{l=2,4 . .}^{\infty} x^{l} P_{l}\left(\frac{1}{\xi}\right)
$$

where introduced functions satisfy following equation $x^{2} \frac{d^{2} S_{2 \nu}(x, t)}{d x^{2}}=Q_{2 \nu}(x, t)$ with conditions $S_{2 \nu}(1, t)=\frac{d S_{2 \nu}(x, t)}{d x}=0$. It is easy to see that a solution of the equation has the form of Mellin convolution:

$$
S_{2 \nu}(x, t)=\int_{x}^{1} \frac{d z}{z}\left(1-\frac{x}{z}\right) Q_{2 \nu}(z, t)
$$

Eventually, performing the summation over $l$ in Eq. (23) we obtain the following expression for amplitude $A_{+}(\xi, t)$ :

$$
\begin{aligned}
\operatorname{Im} A_{+}(\xi, t) & =\xi \frac{\partial}{\partial \xi} \int_{\frac{1-\sqrt{1-\xi^{2}}}{\xi}}^{1} \frac{d x}{x} N_{S}(x, t) \frac{1}{\sqrt{\frac{2 x}{\xi}-1-x^{2}}}, \\
\operatorname{Re} A_{+}(\xi, t) & =\xi \frac{\partial}{\partial \xi} \int_{0}^{\frac{1-\sqrt{1-\xi^{2}}}{\xi}} \frac{d x}{x} N_{S}(x, t)\left[\frac{1}{\sqrt{1-\frac{2 x}{\xi}+x^{2}}}+\frac{1}{\sqrt{1+\frac{2 x}{\xi}+x^{2}}}-\frac{2}{\sqrt{1+x^{2}}}\right] \\
& +\xi \frac{\partial}{\partial \xi} \int_{\frac{1-\sqrt{1-\xi^{2}}}{\xi}}^{1} \frac{d x}{x} N_{S}(x, t)\left[\frac{1}{\sqrt{1+\frac{2 x}{\xi}+x^{2}}}-\frac{2}{\sqrt{1+x^{2}}}\right] .
\end{aligned}
$$


Here in analogy to GPD-quintessence function $N(x, t)$ (6) we introduced its counterpart for twist-3 GPDs:

$$
N_{S}(x, t)=\sum_{\nu=0}^{\infty} x^{2 \nu} S_{2 \nu}(x, t)=\int_{x}^{1} \frac{d z}{z}\left(1-\frac{x}{z}\right) \sum_{\nu=0}^{\infty} x^{2 \nu} Q_{2 \nu}(z, t) .
$$

We see that at twist-3 level the function $N_{S}(x, t)$ provides us with new additional information about forward-like functions $Q_{2 \nu}(x, t)$. Therefore it is important to perform Abel tomography for the twist-3 DVCS amplitude.

\section{Abel tomography and dispersion relations for twist-3 DVCS amplitude}

Now we can obtain the function $N_{S}(x, t)$ if we know the twist-3 DVCS amplitude. We remind that the twist-3 DVCS amplitude given by Eq. (16) in WW approximation can be represented as:

$$
A^{\mathrm{tw} 3}(\xi, t)=A^{\mathrm{tw} 2}(\xi, t)+A_{+}(\xi, t),
$$

where twist-2 amplitude $A^{\text {tw2 }}(\xi, t)$ is given by Eq. (2/3) in terms of GPD-quintessence function $N(x, t)$ and the amplitude $A_{+}(\xi, t)$ by the Eq. (24) in terms of function $N_{S}(x, t)$ (25). Eq. (24) can be brought to Abel type integral equation with help of Joukovski transformation and one can apply the Abel tomography described in the first section to the twist-3 case. The result is the following:

$$
N_{S}(x, t)=\frac{1}{\pi} \frac{\left(1-x^{2}\right)}{\sqrt{1+x^{2}}} \int_{\frac{2 x}{1+x^{2}}}^{1} \frac{d \xi}{\sqrt{\xi}} \frac{1}{\sqrt{\xi-\frac{2 x}{1+x^{2}}}}\left\{\operatorname{Im} A^{\mathrm{tw} 2}(\xi, t)-\operatorname{Im} A^{\mathrm{tw} 3}(\xi, t)\right\} .
$$

Measuring twist-2 and twist-3 DVCS amplitudes (they can be separated studying the azimuthal angle dependence of various DVCS asymmetries) we can access the function $N_{S}(x, t)$ which provides additional information about forward-like functions and GPDs. Using the expression for $\operatorname{Im} A^{\text {tw2 }}$ (2) in terms of the quintessence function $N(x, t)$ we can rewrite the Eq. (27) in the following form:

$$
N_{S}(x, t)=\frac{1-x^{2}}{\sqrt{1+x^{2}}}\left(\int_{x}^{1} \frac{d y}{y} \frac{N(y, t)}{\sqrt{1+y^{2}}}-\frac{1}{\pi} \int_{\frac{2 x}{1+x^{2}}}^{1} \frac{d \xi}{\sqrt{\xi}} \frac{\operatorname{Im} A^{\mathrm{tw} 3}(\xi, t)}{\sqrt{\xi-\frac{2 x}{1+x^{2}}}}\right)
$$

It was shown in Ref. 10 that the dual parameterization representation for the twist-2 DVCS amplitude (2,3) automatically satisfy the once subtracted dispersion relation:

$$
\operatorname{Re} A^{\mathrm{tw} 2}(\xi, t)=2 D(t)+\frac{1}{\pi} v p \int_{0}^{1} d \zeta \operatorname{Im} A^{\mathrm{tw} 2}(\zeta, t)\left(\frac{1}{\xi-\zeta}-\frac{1}{\xi+\zeta}\right)
$$


with the subtraction constant given by the D-form factor (44). This result was obtained recently in Refs. [13, 14] by independent methods. We see that the dual parameterization automatically ensures the dispersion relations for the amplitudes. Now we can repeat the derivation of the dispersion relations for the twist-3 amplitude. To finish this, one has to substitute the expression for $N_{S}(x, t)$ (27) into Eq. (24) for the twist-3 real part of the DVCS amplitude. After simple calculations we obtain once subtracted dispersion relation for the twist-3 DVCS amplitude:

$$
\operatorname{Re} A^{\mathrm{tw} 3}(\xi, t)=2 D(t)+\frac{1}{\pi} v p \int_{0}^{1} d \zeta \operatorname{Im} A^{\mathrm{tw} 3}(\zeta, t)\left(\frac{1}{\xi-\zeta}-\frac{1}{\xi+\zeta}\right) .
$$

We see that for the twist-3 amplitude the subtraction constant is given by the D-form factor, the same as for the twist-2 amplitude.

\section{Conclusions}

We extended the dual parameterization to the case of the twist- 3 contributions to the DVCS amplitude. It is demonstrated that the twist-3 DVCS amplitude in the WandzuraWilczek approximation is determined by single function $N_{S}(x, t)$-the twist-3 analogue of the GPD quintessence function $N(x, t)$-which gives complementary information about forward -like functions and GPDs. We proved dispersion relations for the twist-3 amplitude. It turned out that the subtraction constant for this case is the same as for the twist-2 case, and given by the D - form factor.

We presented equation which directly and unambiguously express GPD quintessence functions $N(x, t)$ and $N_{S}(x, t)$ in terms of twist-2 and twist-3 DVCS amplitudes respectively. Relation of these equations to the Abel transform tomography is elaborated. Amplitudes of hard exclusive processes can be viewed as "photographs" of some object with axial symmetry in plane of variables $X=\sqrt{\frac{2 x}{1+x^{2}}-\xi}$ and $Y=\sqrt{\xi}$. It remains to decipher the deep meaning of these variables and corresponding axial symmetry.

\section{Acknowledgements}

We are thankful to I. Anikin, N. Kivel, D. Müller, K. Semenov-Tian-Shansky, M. Vanderhaeghen and A. Vladimirov for many valuable discussions. The work is supported by the Sofja Kovalevskaja Programme of the Alexander von Humboldt Foundation, and by the Deutsche Forschungsgemeinschaft, the Heisenberg-Landau Programme grant 2007, and the Russian Foundation for Fundamental Research grants No. 06-02-16215 and 07-0291557.

\section{References}

[1] M. V. Polyakov and A. G. Shuvaev, "On 'dual' parameterizations of generalized parton distributions," arXiv:hep-ph/0207153. 
[2] D. Müller, D. Robaschik, B. Geyer, F.M. Dittes, and J. Horejsi, Fortschr. Phys. 42, $101(1994)$;

X. D. Ji, Phys. Rev. Lett. 78 (1997) 610 arXiv:hep-ph/9603249|;

A. V. Radyushkin, Phys. Lett. B 380 (1996) 417 |arXiv:hep-ph/9604317|;

X. D. Ji, Phys. Rev. D 55 (1997) 7114 arXiv:hep-ph/9609381;

J. C. Collins, L. Frankfurt and M. Strikman, Phys. Rev. D 56 (1997) 2982 arXiv:hep-ph/9611433.

[3] K. Goeke, M. V. Polyakov and M. Vanderhaeghen, Prog. Part. Nucl. Phys. 47 (2001) 401 arXiv:hep-ph/0106012|.

[4] M. Diehl, Phys. Rept. 388 (2003) 41 arXiv:hep-ph/0307382.

[5] A. V. Belitsky and A. V. Radyushkin, Phys. Rept. 418 (2005) 1 arXiv:hep-ph/0504030.

[6] S. Boffi and B. Pasquini, "Generalized parton distributions and the structure of the nucleon," arXiv:0711.2625 [hep-ph].

[7] V. Guzey and M. V. Polyakov, Eur. Phys. J. C 46, 151 (2006) arXiv:hep-ph/0507183;

V. Guzey and T. Teckentrup, Phys. Rev. D 74, 054027 (2006) arXiv:hep-ph/0607099.

[8] M. V. Polyakov and M. Vanderhaeghen, "Taming Deeply Virtual Compton Scattering," arXiv:0803.1271 [hep-ph].

[9] N.H. Abel, "Auflösung einer mechanischen Aufgabe”, J. Reine und Angew. Math., 1 (1826) 153;

Abel transform tomography is used in many areas of natural science and engineering, see e.g. the books: A. C. Kak and M. Slaney, "Principles of Computerized Tomographic Imaging", IEEE Press, Inc., New York, 1991; Gorenflo, R. and Vessella, S., "Abel Integral Equations: Analysis and Applications", SpringerVerlag, Berlin-New York, 1991.

[10] M. V. Polyakov, Phys. Lett. B 659 (2008) 542 [arXiv:0707.2509 [hep-ph]].

[11] M. V. Polyakov, "Educing GPDs from amplitudes of hard exclusive processes," arXiv:0711.1820 [hep-ph].

[12] M.V. Polyakov and C. Weiss, Phys. Rev. D 60, 114017 (1999).

[13] O. V. Teryaev, "Analytic properties of hard exclusive amplitudes," arXiv:hep-ph/0510031;

I. V. Anikin and O. V. Teryaev, Phys. Rev. D 76 (2007) 056007 arXiv:0704.2185 [hep-ph]].

[14] M. Diehl and D. Y. Ivanov, Eur. Phys. J. C 52 (2007) 919 [arXiv:0707.0351 [hep-ph]]. 
[15] O. V. Teryaev, Phys. Lett. B 510 (2001) 125 arXiv:hep-ph/0102303.

[16] N.E. Joukovskii, On the problem of cutting vortex filaments, Mat. Sbor. 17 (1895) No 4 702-719 (in Russian);

N.E. Joukovskii, De la chute dans l'air de corps légers de forme allongée, animés d'un mouvement rotatoire, Bull. Inst. Aérodyn. Koutchino (1906) No 1 51-65.

[17] P. A. Vicharelli and W. P. Lapatovich, Applied Physics Letters 50 (1987) 557;

Xian-Fang Li et al, J. Phys. A: Math. Theor. ,40 (2007) 347.

[18] C.K. Chan and P. Lu, J. Phys. A: Math. Gen., 14(1981) 575.

[19] I. V. Anikin, B. Pire and O. V. Teryaev, Phys. Rev. D62 (2000) 071501 hep-ph/0003203.

[20] M. Penttinen, M. V. Polyakov, A. G. Shuvaev and M. Strikman, Phys. Lett. B 491 (2000) 96 arXiv:hep-ph/0006321.

[21] Nucl. Phys. B 589 (2000) 611 |arXiv:hep-ph/0007031|.

hep-ph/0007031.

[22] A. V. Radyushkin and C. Weiss, Phys. Lett. B $493 \quad$ (2000) 332 arXiv:hep-ph/0008214;

A. V. Radyushkin and C. Weiss, Phys. Rev. D $63 \quad$ (2001) 114012 arXiv:hep-ph/0010296.

[23] N. Kivel, M. V. Polyakov and M. Vanderhaeghen, Phys. Rev. D 63 (2001) 114014 arXiv:hep-ph/0012136.

[24] S. Wandzura and F. Wilczek, Phys. Lett. B 72 (1977) 195.

[25] N. Kivel, M. V. Polyakov, A. Schafer and O. V. Teryaev, Phys. Lett. B 497 (2001) 73 arXiv:hep-ph/0007315.

[26] I. V. Anikin and O. V. Teryaev, Phys. Lett. B 509 (2001) 95 arXiv:hep-ph/0102209.

[27] N. Kivel and M. V. Polyakov, Nucl. Phys. B 600 (2001) 334 arXiv:hep-ph/0010150.

[28] M.V. Polyakov, Nucl. Phys. B555, 231 (1999).

[29] P. L. Anthony et al. [E155 Collaboration], Phys. Lett. B 458 (1999) 529 arXiv:hep-ex/9901006;

P. L. Anthony et al. [E155 Collaboration], Phys. Lett. B 553 (2003) 18 arXiv:hep-ex/0204028.

[30] J. Balla, M. V. Polyakov and C. Weiss, Nucl. Phys. B 510 (1998) 327 arXiv:hep-ph/9707515.

[31] B. Dressler and M. V. Polyakov, Phys. Rev. D 61 (2000) 097501 arXiv:hep-ph/9912376. 
[32] D. V. Kiptily and M. V. Polyakov, Eur. Phys. J. C 37 (2004) 105 arXiv:hep-ph/0212372. 